\title{
Estudio de la interpretación a dúo a partir de una propuesta pedagógica para ejercitar la empatía
}

Pablo Dávila Barrio

pablo.klarinetea@gmail.com 


\section{Resumen}

\section{Resumen}

Numerosos estudios reflejan que la empatía desempeña un papel fundamental en la interacción entre los seres humanos. Con este trabajo, se pretende comprobar tales conclusiones a través de una intervención pedagógica en la cual seis participantes de entre 8 y 13 años interpretarán un dúo al principio, realizarán actividades que fomenten la interacción y la empatía durante un periodo de un mes, y volverán a interpretar un dúo similar para finalizar. Los resultados de esta investigación llevada a cabo en el aula de instrumento demuestran que, a pesar de unos resultados ambiguos, existen indicios de que fomentando la empatía y la interacción, la interpretación del discurso musical en dúos se ve positivamente afectada.

Palabras clave: didáctica de la música, empatía, interacción, interpretación a dúo.

\section{Introducción}

Presentamos, en esta publicación, el Trabajo Fin de Máster (TFM) correspondiente al Máster en Didáctica de la Música cursado en la Universidad Jaume I durante el curso académico 2017-2018. Se ha optado por un estudio que precisa de intervención en el aula dentro con un proyecto de investigación-acción. Dicha intervención se ha llevado a cabo durante el periodo de prácticas correspondiente al Máster, que se ejecutó en el Conservatorio Calasancio de Música y Danza de Castellón. Las actividades desarrolladas en el estudio han sido diseñadas a partir de los conocimientos adquiridos durante el año en las otras asignaturas del propio máster. La estructura del trabajo se sustenta sobre dos pilares: la música de cámara y la empatía; se parte de la base de que los elementos indispensables para conseguir una conexión fructífera entre ambos son la interacción y el patrón o los patrones de comportamiento que discurren entre los integrantes de los grupos musicales.

Desde sus comienzos, en el periodo renacentista, durante los siglos XV y XVI, la música de cámara ha sido, en la historia de la música occidental, el género musical de conjuntos instrumentales por excelencia (Hill 2008). Cabe recordar que la orquesta no existía entonces y que el género orquestal siempre se ha basado en los cuartetos de cuerda, la formación instrumental de cámara más importante de todas. Cuando la orquesta no existía como género musical, los compositores, bajo la tranquilidad económica que proporcionaban los grupos adinerados de la sociedad, usaban la 
música de cámara como herramienta para desarrollar sus aptitudes y habilidades compositivas, por lo que podemos afirmar que este tipo de música siempre ha estado a la vanguardia en Occidente (Burkholder, Grout y Palisca 2015).

En el cuerpo legislativo del sistema educativo español, la práctica de la música de cámara se justifica al entenderla como un proceso que «responde a un conjunto de necesidades del alumnado de música que difícilmente pueden ser atendidas si no es a través de esta actividad.» (Real Decreto 1577/2006, 2865) ${ }^{1}$. Está concebida como una asignatura complementaria de la formación instrumental: «el cultivo de la música de cámara resulta absolutamente complementaria de la formación instrumental, permitiendo la aplicación práctica de los conocimientos adquiridos en la clase de instrumento, dentro de una actividad que, a causa de su carácter lúdico, permite la práctica musical en condiciones ideales de espontaneidad y distensión» (Real Decreto 1577/2006, 2866).

En cuanto a la empatía, tiene un papel fundamental en la interacción entre los seres humanos. La empatía es el mayor contribuyente al fortalecimiento de la interacción social gracias a su capacidad para motivar a las personas a cooperar, compartir recursos y ayudar a otros (de Vignemont y Singer 2006; Hosking y Fineman 1990; Van Lange 2008). También sostenemos que la empatía es una herramienta capital en la promoción del comportamiento prosocial a través del aumento de acciones positivas, de ayuda y reflexivas (Gano-Overway et al. 2009; Natale y Sora 2010). Por lo tanto, encarna una cualidad determinante en el desarrollo de la comunicación. Sin embargo, el valor de la empatía y su importancia social no son promovidos lo suficiente en las aulas de educación en España. (Gorroño Cortázar 2014). De hecho, de todos los participantes en este trabajo de interacción, solo dos de ellos tenían algunas nociones sobre empatía.

Consecuentemente, este trabajo propone un método pedagógico a través del cual el aprendizaje de la asignatura de cámara adopta un enfoque innovador $y$ ofrece una alternativa educativa. Con el presente proyecto de intervención se plantea la hipótesis de que trabajando la empatía, al influir en la capacidad de interacción entre los seres humanos y en su mejora, la interpretación del discurso musical se enriquece. Así, nos fijamos los siguientes objetivos: fomentar la empatía a través del aprendizaje de los dúos, desarrollar la capacidad de interactuar para conseguir un objetivo común, y emplear en el aula una estrategia pedagógica donde la comunicación y la creatividad musical estén incluidas en la práctica docente.

Muchos autores han comprobado la habilidad que tiene la empatía para potenciar las aptitudes sociales así como la influencia que tiene en el proceso de aprendizaje -Goleman, 3 lacoboni (2009) y Rabinowitch (2009) entre otros. Lo que sostenemos es que tanto la 
práctica de la música de cámara a dúo como el desarrollo de la empatía obtienen múltiples beneficios mutuos. La práctica de la música de cámara a dúo nos brinda la posibilidad de enseñar los componentes básicos de la música de cámara: aprender a dirigir, seguir, unir el tono, mezclar el sonido, mostrar ritmos preparatorios, respirar juntos y otros gestos no verbales. Wetzel (2007) menciona que la práctica de dúos contribuye al desarrollo de parámetros musicales tales como la conciencia del pulso y la entonación. En consecuencia, los dúos se erigen como una excelente herramienta para desarrollar ciertas habilidades, como la lectura a primera vista. Además, refuerza el concepto de la música como una actividad social y aumenta el interés de los estudiantes, ya que a la interpretación musical se añade la relación entre compañeros.

\section{Objetivos}

Estos son los objetivos que se proponen en la intervención pedagógica:

- Fomentar la empatía a través del aprendizaje interpretativo de los dúos.

- Desarrollar la capacidad de interactuar para conseguir un objetivo común.

- Emplear en el aula una estrategia pedagógica donde la comunicación y la creatividad musical estén incluidas en la práctica docente.

- Examinar, constatar y analizar los cambios emocionales e interpretativos después de realizar ejercicios que fomentan la interacción entre personas y la empatía.

- Aprender algunos de los elementos y habilidades necesarias para interpretar una obra musical en conjunto.

\section{Material y método}

En este apartado se presenta la propuesta de intervención pedagógica descrita con la finalidad de verificar la hipótesis que planteamos al inicio del trabajo. Para ello se ha seguido el siguiente procedimiento metodológico:

1. Revisar y analizar la bibliografía existente hasta el momento sobre la empatía y la interpretación a dúo, así como la referida a la importancia que adquiere la empatía en la relación entre personas que interactúan para conseguir un objetivocomún.

2. Efectuar una intervención de carácter pedagógico en un aula de música, en este caso de clarinete, planeada y basada en una exploración realizada previamente. 
3. Realizar una evaluación cualitativa del proceso de intervención, una reflexión crítica sobre él y la elaboración de propuestas de mejora del proceso desarrollado.

Así pues, antes de realizar la intervención pedagógica se repasa y analiza la bibliografía, creando un marco teórico. En un primer apartado, el marco teórico del trabajo comienza con la revisión de la normativa española en materia de educación que hace referencia a la necesidad de formar a los alumnos emocional, cognitiva y afectivamente, así como a la importancia que tiene para dicha formación la interacción y la comunicación entre seres humanos. En este sentido se hallan numerosas alusiones a los conceptos de respeto y tolerancia, entendidos como herramientas de las que se ha de dotar a los alumnos para solucionar de manera pacífica los problemas en las relaciones del día a día.

Tanto en la Ley Orgánica 2/2006, de 3 de mayo (LOE) ${ }^{1}$ como en el Real Decreto ${ }^{2}$ 1577/2006, de 22 de diciembre, se pueden encontrar varios artículos que muestran cómo de necesaria es la educación emocional para, por ejemplo, afianzar el espíritu emprendedor con actitudes de creatividad, flexibilidad, iniciativa, trabajo en equipo, confianza en uno mismo y sentido crítico. (Ley Orgánica 2/2006, artículo 33) o conocer los valores de la música y optar por los aspectos emanados de ella que sean más idóneos para el desarrollo personal», así como en el artículo 3 sobre objetivos específicos: "Formar una imagen ajustada de las posibilidades y características musicales de cada uno, tanto a nivel individual como en relación con el grupo, con la disposición necesaria para saber integrarse como un miembro más del mismo o para actuar como responsable del conjunto» el Real Decreto 1577/2006.

El segundo apartado del marco teórico se centra en la empatía. La empatía se suele explicar con expresiones como estas: "meterse en la piel del otro» o "ponerse en la situación del otro». Sin embargo, muchos son los investigadores que han intentado dar una definición más rigurosa, completa y acertada del concepto de empatía: "La empatía es un proceso o procedimiento mediante el cual una persona imagina los pensamientos, sentimientos $y$ emociones de otra persona»; dicho de otro modo, la empatía es la capacidad de los seres humanos para sentir las experiencias de los demás como propias (Clark, 1980; Cohen y Strayer 1996; Hoffman 2001).

En 1995, Daniel Goleman publicó sus teorías en el libro Emotional Intelligence, la empatía se ha clasificado en tres tipos diferentes: la empatía cognitiva, la empatía emocional y la empatía compasiva. La primera hace referencia a la adopción del punto de vista de la otra persona para comprender tanto su situación como sus

\footnotetext{
${ }^{1}$ BOE, consultado enhttps://boe.es/buscar/doc.php?id=BOE-A-2007-1221el $16 / 05 / 2018$.

2 BOE, consultado enhttps://boe.es/buscar/doc.php?id=BOE-A-2007-1221el16/05/2018.
} 
emociones y sentimientos. La segunda consiste en que uno sienta lo que la otra persona siente. Por último, la tercera tiene que ver con percibir que la otra persona necesita ayuda $y$, consecuentemente, ofrecérsela de manera espontánea e incondicional.

Posteriormente, aventuramos una aproximación al campo de la neurociencia. La neurociencia es un campo de la ciencia que estudia el sistema nervioso junto con todos sus aspectos y funciones. Gracias al estudio realizado con monos macacos por el italiano Rizzolatti en 1996, se descubrió que un grupo de neuronas de la corteza premotora del macaco no solo se "encendían» cuando el animal ejecutaba ciertos movimientos, sino que, simplemente con contemplar a otros hacerlo, ya fuera otro animal o un ser humano, también se activaban. Se las llamó «neuronas espejo» o «especulares».

A raíz del descubrimiento de las neuronas espejo, es imposible no objetar sobre el papel de la imitación en el ámbito empático. En los últimos tiempos, se ha producido una convergencia entre los modelos cognitivos de imitación, los relatos de psicología social sobre su penetración y sus vínculos funcionales tanto con la empatía como con el gusto, y los descubrimientos de la neurociencia de los mecanismos neuronales de la imitación y la empatía. Esta convergencia crea un marco sólido en el que la teoría y los datos empíricos se refuerzan mutuamente.

El tercer y último apartado del marco teórico se centra en la interacción y comunicación de los seres humanos enfocado a partir de la interpretación musical. La creación o la interpretación conjunta de música es un poderoso medio social y emocional. La música ha servido como un medio para ciertos tipos de interacción humana, muy probablemente desde los albores de la humanidad. Las interacciones entre los humanos constituyen un componente integral de la ceremonia y el ritual en todas las culturas, así como una forma significativa de arte y entretenimiento. Estos tipos de interacciones se suelen caracterizar por un nivel relativamente alto de significación social y emocionalidad. Una interpretación o creación que conlleve una acción con otra persona requiere de la interacción para que esta sea fructífera.

Rabinowitch, Cross y Burnard (2011) presentan el concepto de la intersubjetividad, que representa el intercambio de intenciones, emociones y procesos cognitivos presentes en una interacción. Como se muestra en ese estudio, la intersubjetividad consta de cuatro apartados: movimiento, emocionalidad, sincronización y confianza.

$\mathrm{Si}$ estos conceptos los estudiamos y analizamos en un grupo de cámara, observaremos que los aspectos interactivos que se suceden en la relación son los mismos. Sin embargo, al estar dentro de un contexto temporal, característica inherente a una interpretación musical, se engloban dentro de un mismo parámetro: el movimiento. 
A la hora de comunicarse entre los integrantes de un conjunto instrumental, el movimiento es una herramienta que permite coordinar y sincronizar las intenciones musicales de cada individuo del conjunto Las funciones del movimiento en una interpretación son muy variadas, desde gestos y contactos visuales hasta señales acústicas como cambios de tempo y de volumen. Nuestro cuerpo transmite constantemente información sensible sobre nuestras intenciones, sentimientos y personalidad. Incluso cuando estamos quietos o en silencio, los gestos, las posturas, las expresiones faciales y la apariencia hablan por nosotros, y pueden resultar muy elocuentes. Al interpretar una composición por parte de un grupo de música de cámara, el uso de diferentes movimientos o gestos tiene dos objetivos principales: la sincronización y la búsqueda de un hilo expresivo común entre los integrantes del grupo.

Para concluir con el marco teórico, se hace referencia al poder expresivo que tiene el movimiento. El movimiento también influye en la expresión del discurso musical. Por ejemplo, una señal de entrada puede ofrecer la posibilidad de dar más información que meramente la coordinación del conjunto, puede también mostrar el carácter, el tempo y la dinámica del discursomusical.

Frith (1996) explica que en una interpretación se alude las siguientes acciones y reflexiones: la primera sería la ocasión misma de poder interpretar una pieza (por ejemplo, posturas y gestos específicos); la segunda, la música que se interpreta (el contenido narrativo del material musical), junto con los verdaderos estados del ejecutante individual (estado de ánimo actual, sentido del yo, etc.). Por lo tanto, esta explicación sugiere que existe una correlación entre la información contenida en la música (su propio contenido narrativo), el estado real del artista intérprete o ejecutante (los artistas individuales en el escenario, con sus propias personalidades) y las conductas en las que pueden participar para presentar tanto la música como a ellos mismos a sus compañeros de trabajo y audiencia. Dicho esto, la manera de hacer llegar estas reflexiones al oyente y a los músicos con los que uno está interactuando no es otra que a través del movimiento y su expresión.

Finalmente, Davidson (2012) concluye que los seres humanos dependemos de las expresiones faciales para la comunicación de la intención emocional en una reunión social: la cara revela claves vitales sobre el estado emocional de la persona que tenemos delante. En música ocurre de la misma manera. Sin embargo, en los instrumentos de viento y particularmente en el clarinete, la cara está demasiado ocupada con el control del instrumento, por lo que el dominio del movimiento corporal es esencial para la expresión que el intérprete quiere otorgar a su discurso musical.

En consecuencia, las habilidades de ejecución musical involucran los aspectos biomecánicos que conlleva tocar la música con fluidez, 
que a su vez coexisten con intenciones expresivas manifestadas a través de movimientos corporales y expresiones faciales que permiten la comunicación de la intención musical (aclarar rasgos estructurales musicales) o el significado (narrativa designada como Liszt trabajo de piano).

Una vez encuadrado y sustentado el trabajo en estos parámetros y expuestos los conceptos teóricos básicos, expondremos en que consistió la intervención pedagógica en el aula, en el propio campo de trabajo.

Los alumnos que participan en este proyecto son todos alumnos del Conservatorio Calasancio y están bajo la tutela del profesor de clarinete del centro: Rafa Palomar. En total son seis niños/adolescentes que cursan desde segundo de Grado Elemental hasta primero de Grado Profesional, de edades comprendidas entre los 8 y los 14 años. La pareja A está compuesta por los alumnos 1 y 2. Ambos alumnos tienen 8 años de edad y cursan 20 de Grado Elemental. La pareja B está compuesta por los alumnos 3 y 4 . Ambos alumnos cursan 4음 de Grado Elemental. Sin embargo, tienen edades diferentes, 14 y 13 años, respectivamente. La pareja C está compuesta por los alumnos 5 y 6, quienes cursan 10 de Grado Profesional y 40 de Grado Elemental, respectivamente. El alumno 5 tiene 12 años y el alumno 6 tiene 13 años; al primero de ellos se le ha adelantado un curso en las enseñanzas de música.

Cada pareja trabajará 2 dúos de características similares. Al principio del proyecto interpretarán dos y al final de la propuesta de intervención tocarán los dos restantes y se compararán ambas interpretaciones musicales. La pareja A interpretará Bagatelle de Schumann y St. Anthony Chorale de J. Haydn; la pareja B, Nine progressive duets in 6/8 y St. Anthony Chorale de J. Haydn, y la pareja C interpretará Melody de Schumann y Tema de la sinfonía del Nuevo de Dvorak.

\subsection{Metodología}

La metodología utilizada en esta intervención se caracteriza por basarse en el aprendizaje cooperativo y participativo. La música, así como los sentimientos y la capacidad para expresarlos, requiere más de la práctica que de la teoría para su ejercicio y puesta en práctica. El principal objetivo de estas actividades es el desarrollo integral del alumno. Los alumnos tendrán que utilizar los conocimientos adquiridos sobre lo que es la empatía y comenzar a emplear aquellas herramientas que se les proporcionan para la mejor interpretación de una obra con un compañero, como pueden ser la imitación, la escucha activa o lacreatividad.

Ejercicio de entrevista: Este ejercicio, presentado por Scott Tink, consiste en que los alumnos recreen una entrevista, como si de una entrevista en televisión se tratara, en la que el anfitrión o 
entrevistador plantea una situación y sugiere una acción empática al invitado. El objetivo es que el anfitrión obtenga respuestas empáticas al extraer ideas del invitado sobre algunas de sus experiencias, sentimientos y actitudes asociadas al escenario.

Ejercicio de mímica: Este ejercicio, llevado a cabo en diferentes investigaciones (Cross, Laurance y Rabinowitch 2012), consiste en adivinar los sentimientos o situaciones emocionales que el compañero está describiendo a través de la mímica. Para ello, los alumnos usaran la comunicación no verbal como herramienta para interactuar. El ejercicio se divide en dos partes, en la primera, los alumnos trabajarán con sentimiento; en la segunda, con situaciones emocionales.

Ejercicio de eco: Este ejercicio, presentado en Gorroño-Cortazar (2014) consiste en que un alumno repita lo que el otro sugiere usando la escucha activa, la capacidad de memorización y la imitación para conseguirlo. El ejercicio se dividirá en dos apartados: el rítmico y el melódico. En el primero, se trabajará únicamente con ritmos y en el segundo se añadirá el componente melódico.

Ejercicio de composición: Este ejercicio, aplicado por Rabinowitch (2012), consiste en componer conjuntamente con el compañero de dúo una pequeña canción y crear una historia relacionada. Esta constará de 16 compases y cada alumno compondrá 8. Tendrá que aprender también los 8 compases del compañero para posteriormente interpretarlos ambos a la vez. Una vez creada la historia, los alumnos intentarán ponerle música entre los dos, teniéndose que comunicar e intercambiar opiniones, según los sentimientos o situaciones que sugieran la historia y ellos mismos.

\subsection{Diseño y temporalización}

Tabla 1. Diseño y temporalización de la intervención pedagógica

\begin{tabular}{|l|l|l|l|l|}
\hline \multirow{2}{*}{ Sesiones } & $\begin{array}{l}\text { Fecha (año } \\
\text { 2018) }\end{array}$ & Actividad & Contenido & Temporalización \\
\hline \multirow{2}{*}{ Sesión 1 } & $2-3$ de mayo & $\begin{array}{l}\text { Interpretación } \\
\text { dúo }\end{array}$ & Primera interpretación & 20 minutos \\
\cline { 3 - 5 } & & $\begin{array}{l}\text { Introducción a } \\
\text { la materia }\end{array}$ & $\begin{array}{l}\text { Explicación empatía y } \\
\text { proyecto }\end{array}$ & 10 minutos \\
\cline { 3 - 6 } & & Entrevista TV & Primera fase & 15 minutos \\
\hline \multirow{2}{*}{ Sesión 2 } & $\begin{array}{l}8-9-10 ~ d e \\
\text { mayo }\end{array}$ & Entrevista TV & Segunda fase & 10 minutos \\
\cline { 3 - 6 } Sesión 3 & $\begin{array}{l}15-16-17 \text { de } \\
\text { mayo }\end{array}$ & ECo & Ambas fases & 30 minutos \\
\hline \multirow{2}{*}{ Sesión 4 } & $\begin{array}{l}22-23-24 \text { de } \\
\text { mayo }\end{array}$ & Composición & Apartado melódmico & 25 minutos \\
\hline \multirow{2}{*}{ Sesión 5 } & $\begin{array}{l}29-30-31 \text { de } \\
\text { mayo }\end{array}$ & Repaso & $\begin{array}{l}\text { Recordar el proceso } \\
\text { Ilevado a cabo }\end{array}$ & 15 minutos \\
\cline { 3 - 6 } & & Interpretar dúo & Interpretación final & 20 minutos \\
\hline
\end{tabular}




\subsection{Criterios de evaluación}

- Se establecen los siguientes puntos y momentos que evaluar durante el proyectode intervención:

- Se hace una evaluación inicial o diagnóstica sobre la características empáticas de los alumnos.

- Se hace una evaluación inicial sobre la interpretación de un dueto.

- Se hace una evaluación final sobre las características empáticas de los alumnos.

- Se hace una evaluación final sobre la interpretación de un dueto.

Evaluación inicial y final sobre las características empáticas: En este proyecto, la evaluación inicial se ha llevado a cabo a través de cuestionarios. Aunque se entiende como uno general, varía en el tipo de preguntas, algunas abiertas y otras cerradas. El cuestionario utilizado ha sido el que emplea Ainhoa Gorroño Cortázar en propuesta musical para el desarrollo de la empatía en alumnos de 11 y 12 años (2014).

Al final del proceso de intervención en el aula, se efectuará la evaluación final, tan necesaria pero observar el cambio, si es que ha sucedido, respecto al punto de partida. Con la finalidad de ser lo más transparente e imparcial posible, el método de evaluación inicial será idéntico al de la evaluación final.

CRITERIOS DE EVALUACIÓN DEL CUESTIONARIO: El cuestionario de Ainhoa Gorroño Cortázar empleado en Propuesta musical para el desarrollo de la empatía en alumnos de 11 y 12 años (2014) está compuesto por 42 ítems, de los cuales 32 son cerrados y 10 abiertos. Respecto a los ítems cerrados tienen cinco calificaciones distintas, que a su vez indican la capacidad empática.

En cuanto a los ítems abiertos, se evaluará la cantidad y variedad de emociones y sentimientos descritos, es decir, cuantas más emociones y variedad se presenten, más posibilidades hay de que la persona sea empática.

Tabla 2. Equivalencia de los niveles empáticos

\begin{tabular}{|l|l|l|}
\hline & Calificación & Capacidad \\
\hline 1 & Nunca & Nada empático \\
\hline 2 & Raramente & Poco empático \\
\hline 3 & Algunas veces & Algo empático \\
\hline 4 & Frecuentemente & empático \\
\hline 5 & Muy frecuentemente & Muy empático \\
\hline
\end{tabular}

Evaluación de la interpretación del dueto: El método de evaluación para la interpretación del dueto se realizará a través de 
una rúbrica creada por el autor de este trabajo. Una rúbrica es un instrumento para realizar una evaluación alternativa y auténtica del desempeño de los estudiantes. Se genera mediante un listado de un conjunto de criterios específicos y fundamentales que permiten apreciar el aprendizaje, así como las competencias logradas por los estudiantes en una materia en concreto.

Criterios de evaluación de la rúbrica: Los criterios de evaluación y calificación establecidos en la rúbrica han sido elaborados tras un proceso largo de aprendizaje por parte del autor de este trabajo. Autores y docentes coinciden en que la principal razón por la que una rúbrica no es buena es porque tanto los criterios de evaluación como sus calificaciones no están bien reflexionadas y manifestadas (Tierney y Simon 2004), (Reddy 2005; Andrade 2010, Popham 1997). El problema principal es la validez y fiabilidad de estos dos aspectos.

Con la finalidad de disponer de una rúbrica fiable, una vez creada, la he ejecutado en clase, la he modificado y la he consultado con otros docentes y compañeros antes de conseguir la versión óptima que permitirá evaluar lo más objetivamente posible.

\section{Resultados}

En términos generales, no se puede afirmar que se haya cumplido la hipótesis planteada al principio del trabajo, en la que decididamente postulábamos que aquellos ejercicios en el aula que consiguieran mejorar la relación empática y emocional entre los miembros de un dúo de clarinetes repercutirían de inmediato en una mejora sistemática de la calidad interpretativa y musical de dicho dúo. Los resultados obtenidos han sido contradictorios, discordantes e incluso, en algunos casos, paradójicos, aunque algunos de ellos resultan esperanzadores.

\section{Resultados iniciales}

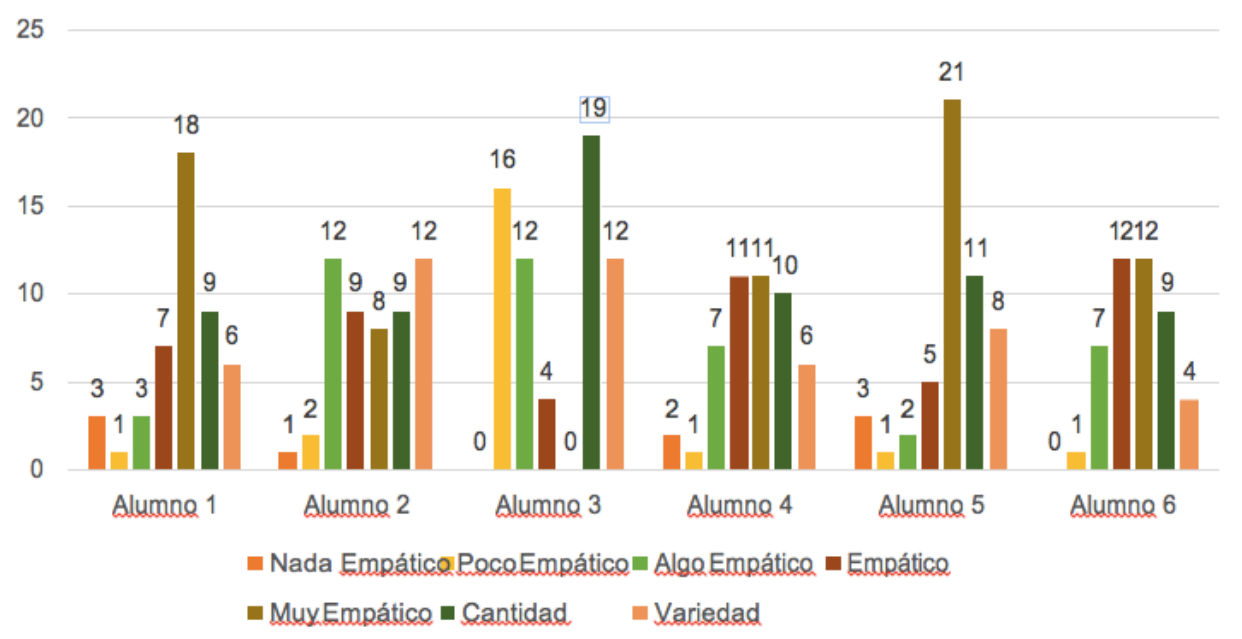

Figura 1. Resultados iniciales del cuestionario 
Son constatables las diferencias que se han observado en los cuestionarios iniciales en comparación con los cuestionarios finales. Es lógico pensar que, tras un periodo de trabajo centrado en la mejoría de un aspecto concreto, los resultados sean al menos ligeramente mejores, como mínimo. Sin embargo, los datos de los resultados finales no muestran una clara mejoría en los alumnos.

\section{Resultados finales}

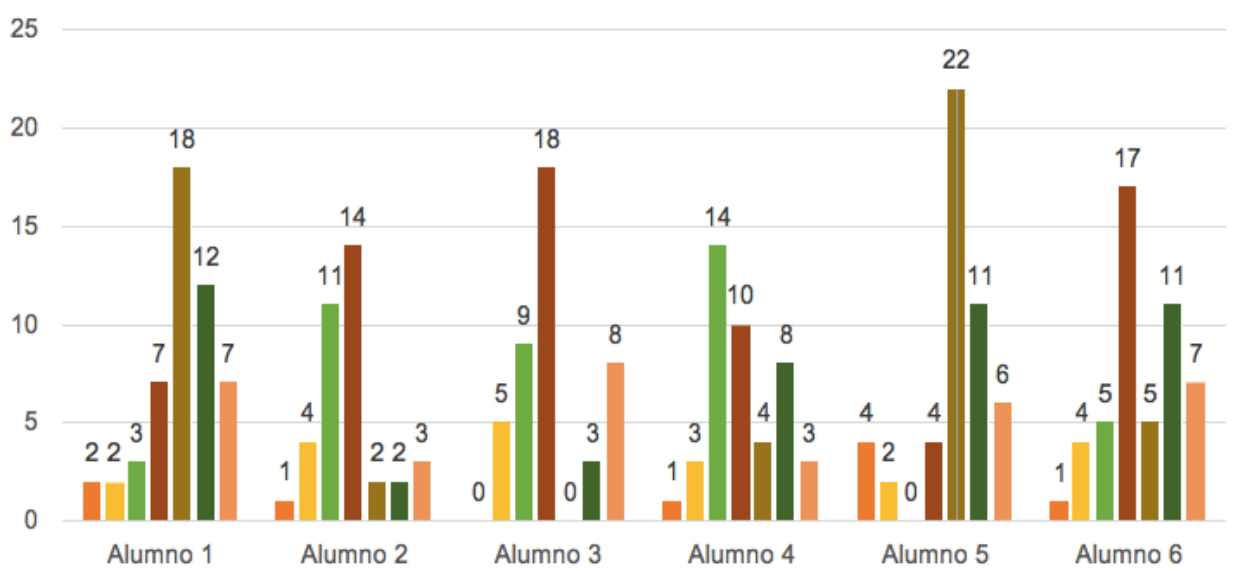

Figura 2. Resultados finales del cuestionario

Si comparamos las dos tablas de resultados, es significativo el hecho de que los extremos de resultados finales dan valores más bajos, y quedan más explícitos los niveles empáticos de los participantes. Además, los resultados que hacen referencia a los cuestionarios con preguntas abiertas muestran un aumento en la variedad y cantidad de respuestas empáticas. Esto nos permite afirmar que las actividades propuestas y realizadas han modificado, si no sustancialmente, sí al menos según una tendencia clara, el comportamiento de los alumnos, quienes muestran mayor criterio a la hora de identificar el significado de las emociones y las situaciones empáticas.

En cuanto a las calificaciones obtenidas a través de la rúbrica, se observa que en el apartado comunicativo las puntuaciones experimentan una notable mejoría tras haber realizado las actividades, por lo que se confirma que sí ha tenido un efecto positivo traducido en una mejora de la interacción durante el discurso musical.

Tabla 3. Calificación inicial rúbrica interpretación

\begin{tabular}{|c|c|c|c|c|}
\hline & & Pareja A & Pareja B & Pareja C \\
\hline \multirow{2}{*}{$\begin{array}{c}\text { Appartado } \\
\text { interpretativo }\end{array}$} & Pulso & 1,5 & 0,8 & 1,2 \\
\cline { 2 - 5 } & Dinámica & 0,4 & 0,5 & 1 \\
\hline
\end{tabular}




\begin{tabular}{|c|c|c|c|c|}
\hline & Articulación & 0,7 & 1 & 1 \\
\cline { 2 - 5 } & Musicalidad & 0,7 & 0,5 & 0,6 \\
\hline \multirow{4}{*}{$\begin{array}{c}\text { Apartado } \\
\text { comunicativo }\end{array}$} & Sincronización & 1,5 & 1 & 1,5 \\
\cline { 2 - 5 } & Roles & 0,5 & 0,7 & 0,7 \\
\cline { 2 - 5 } & Interacción & 0,7 & 0,6 & 1 \\
\hline \multirow{2}{*}{} & TOTAL & 6 & 5,1 & 7 \\
\hline
\end{tabular}

Tabla 4. Calificación final rúbrica interpretación

\begin{tabular}{|c|c|c|c|c|}
\hline & & Pareja A & Pareja B & Pareja C \\
\hline \multirow{4}{*}{$\begin{array}{c}\text { Appartado } \\
\text { interpretativo }\end{array}$} & Pulso & 1,5 & 1 & 1,5 \\
\cline { 2 - 5 } & Dinámica & 1 & 0,5 & 0.8 \\
\cline { 2 - 5 } & Articulación & 1 & 1 & 1 \\
\cline { 2 - 5 } & Musicalidad & 0,7 & 0,5 & 0,8 \\
\hline \multirow{3}{*}{$\begin{array}{c}\text { Appartado } \\
\text { comunicativo }\end{array}$} & Sincronización & 1,8 & 1,5 & 1,8 \\
\cline { 2 - 5 } & Roles & 0,8 & 1 & 1 \\
\cline { 2 - 5 } & Interacción & 1,5 & 1,5 & 1,5 \\
\hline & TOTAL & 8,3 & 7 & 8,4 \\
\hline
\end{tabular}

Por otro lado, en la interpretación a dúo intervienen factores externos (como el propio instrumento) que pueden determinar la calidad de la interacción, y es muy probable que estos resultados estén algo desviados por el acierto o desacierto de la elección de las actividades en sí, aparte de la renuncia a medir otros parámetros que no se han trabajado o tenido en cuenta.

\section{Conclusiones}

Se podría considerar que, a través de la realización de las actividades que fomentan la empatía y la interacción, la interpretación de un dueto se ve afectada positivamente. Sin embargo, los dispares resultados obtenidos en los cuestionarios no indican lo mismo. Por ello, verdaderamente, no se puede dictaminar que la hipótesis se haya probado completamente.

Las razones para que se dé este contraste entre unos resultados y otros pueden obedecer a factores como la concentración y atención de los alumnos al realizar los cuestionarios, la falta de costumbre de contestar cuestionarios de este tipo, la mayor o menor experiencia del alumnado interpretando dúos, las limitaciones y obstáculos tanto musicales como instrumentales que han mostrado los alumnos, y, por supuesto, las pocas sesiones efectuadas. 
Andrade, Heidi Goodrich. 2005. «Teaching with Rubrics: The Good, the Bad, and the Ugly». College Teaching 53 (1): 27-30.

Blakemore, Sarah-Jane y Jean Decety. 2001. «From the Perception of Action to the Understanding of Intention». Nature Reviews Neuroscience 2 (8): 561-567.

Burkholder, J. Peter, Donald J. Grout, y Claude V. Palisca. 2015. Historia de la música occidental. Madrid: Alianza Música.

Davidson, Jane W. 2012. "Bodily movement and facial actions in expressive musical performance by solo and duo instrumentalists: Two distinctive case studies». Psychology of Music 40 (5): 595-633.

Frith, Simon. 1996. Performance rites: Evaluating popular music. Oxford: Oxford University Press.

Gano-Overway, L., M. Newton, T. Magyar, M. Fry, M. Kim, y M. Guivernau. 2009. "Influence of caring youth sport contexts on efficacy-related beliefs and social behaviours». American Psychological Association 45 (2): 329-340.

Goebl, Werner y Caroline Palmer. 2009. «Synchronization of timing and motion among performing musicians». Music Perception 26 (5): 427-438.

Gorroño Cortázar, Ainhoa. 2014. Propuesta musical para el desarrollo de la empatía en alumnos de 11 y 12 años. Logroño: Universidad Internacional de La Rioja.

Haddon, Elizabeth y Mark Hutchinson. 2015. «Empathy in Piano Duet Rehearsal and Performance». Empirical Musicology Review 10 (2): 140-153.

Hill, John Walter. 2008. La música barroca. Nueva York: Akal.

Hosking, Dian y Stephen Fineman. 1990. «Organizing processes». Journal of Management Studies 27 (6): 583-604.

lacoboni, Marco. 2009. "Imitation, Empathy, and Mirror Neurons». The Annual Review of Psychology 60: 653-670

Keysers, Christian. 2009. "Mirror Neurons». Current Biology 19 (21): 971-973.

Mota, Davi, Maurío Loureiro y Rafael Laboissière. 2013. «Analysis of synchronization patterns in clarinet duos using acoustic and kinematic parameters». Nuestro cuerpo. Nuestra música 1 (1): 199-206. 
Natale, Samuel Michal y Sebastian A. Sora. 2010. «Ethics in Strategic

Thinking: Business Processes and the Global Market Collapse». Journal of Business Ethics 94: 309-316. doi.org/10.1007/s10551009-0270-2.

Pierson, Wetzel. 2007. The pedagogical benefits of duet playing. A Vannetelbosch companion. Columbus, $\mathrm{OH}$ : The Ohio State University.

Popham, William James. 1997. "What's Wrong -and What's Rightwith Rubrics». Educational Leadership 55 (2): 72-75.

Rabinowitch, Tal-Chen. 2012. "Musical games and empathy». Education and Health 30 (3): 80-84.

Rabinowitch, Tal-Chen, lan Cross y Pamela Burnard. 2011. «Longterm musical group interaction has a positive influence on empathy in children». Psychology of Music 41 (4): 484-498.

Reddy, Y. Malini y Heidi Andreade. 2010. Assessment \& Evaluation in Higher Education 35 (4): 435-448.

Rizzolatti, Giacomo y Laila Craighero. 2004. "The Mirror-Neuron system». Annual Review of Neuroscience 27: 169-192.

Tierney, Robin y Marielle Simon. 2004. "What's still wrong with rubrics: focusing on the consistency of performance criteria across scale levels». Practical Assessment, Research and Evaluation 9 (2).

Sebanz, Natalie, Harold Bekkering y Günther Knoblich. 2006. «Joint Action: Bodies and Minds Moving Together». Trends in Cognitive Sciences 10 (2): 70-76.

Van Lange, Paul A. M. 2008. "Does empathy trigger only altruistic motivation? How about selflessness or justice?» Emotion 8 (6): 766-774. 